\title{
Evidence that the Production of Swollen Bodies in the Dienes Phenomenon may be Confined to One of Two Interacting Strains of Proteus mirabilis
}

\author{
By J. WOLSTENHOLME* \\ Department of Bacteriology, University of Birmingham, Birmingham, B $152 T J$
}

(Received 13 October 1972)

The Dienes phenomenon occurs when two Proteus swarms meet, causing mutual inhibition and the formation of large sphaeroplast-like bodies at the zone of interaction. This phenomenon was first described by Dienes (1946). Dienes (1947) concluded that all strains were capable of producing viable large bodies; in any combination of two strains tested, these always appeared, on serological and other grounds, to be identical with only one of the strains involved. As only about $50 \%$ of visible bodies were viable, he concluded that the non-viable bodies were derived from the other strain.

To test Dienes's conclusion the two interacting strains were separated by a membrane produced (as for electron microscopy) by dropping $2 \%(\mathrm{w} / \mathrm{v})$ collodion solution in amyl acetate on the surface of water in a $15 \mathrm{~cm}$ dish. The membrane did not appear to affect the nature of the phenomenon, and it became apparent that, whereas the formation of large bodies was induced, it was an asymmetrical phenomenon, in that a very large proportion of the bodies appeared upon one of the two strains.

Four strains of Proteus mirabilis (H, M, 24, OI I) were isolated from pathological material in the laboratory of the Queen Elizabeth Hospital, Birmingham, and were identified by their biochemical reactions. They produced typical lines of inhibitory demarcation, one with another, on culture; they did not produce colicines and had all been subcultured at least six times before examination.

Swarmers were produced by inoculation of one edge of an agar plate and incubating overnight at room temperature $\left(25^{\circ} \mathrm{C}\right)$, so that no temperature changes occurred during examination, that might affect large body production (Dienes, 1946). Swarmers were then removed from the circumference of the colony with a fine glass spreader, for inoculation. These were always taken from cultures at least $15 \mathrm{~h}$ old (Šourek, I968).

A microscope slide was set in an agar plate and a sample of one of the swarmers was inoculated on the agar over the centre of the slide. A collodion membrane was produced, carefully laid over the swarmers, and the folds teased out. The swarmers of the second strain were emulsified on a coverslip, with a drop of nutrient broth and this was placed over the first strain. It was important that both layers should be of the same density, and not too dense, as a thick growth tended to obscure the reaction. The preparation was cut out with a hot wire and scalpel and observed under a phase contrast microscope, the two layers being in different focal planes.

Each system was tested with the strains in both upper and lower positions, and results were read after $3 \mathrm{~h}$ at $25{ }^{\circ} \mathrm{C}$. Strain $\mathrm{H}$ produced large bodies in reaction with $\mathrm{M} ; 24$ produced them with or I. In both cases, the degree of transformation was greater, up to $50 \%$ of the

\footnotetext{
* Present address: Department of Microbiology, University of Birmingham, Birmingham, B I5 2 TT.
} 
swarmers in the inoculum, when $\mathrm{H}$ and 24 occupied the upper positions, whereas $\mathrm{M}$ and $\mathrm{OI}$ failed to produce more than about $\mathrm{I}$ or $2 \%$ in either position. As in the case of Dienes's strains, about $50 \%$ of these bodies were viable on subculture.

This confirms Dienes's observation that viable bodies belong to only one of two reacting strains, but casts doubt on his belief that the non-viable bodies belonged to the other strains. It also appears that immediate contact between the reacting strains is not essential, and this decreases the probability that the process is analogous to direct conjugation.

\section{REFERENCES}

Dienes, L. (1946). Reproductive processes in Proteus cultures. Proceedings of the Society for Experimental Biology and Medicine 63, 265-270.

DiENES, L. (1947). Further observations on the reproduction of bacilli from large bodies in Proteus cultures. Proceedings of the Society for Experimental Biology and Medicine 66, 97-98.

ŠOUREK, J. (I968). On some findings concerning Dienes phenomenon in swarming Proteus strains. Zentralblatt für Bakteriologie, Parasitenkunde, Infektionskrankheiten und Hygiene (Abteilung I. Originale) 208, 419-427. 